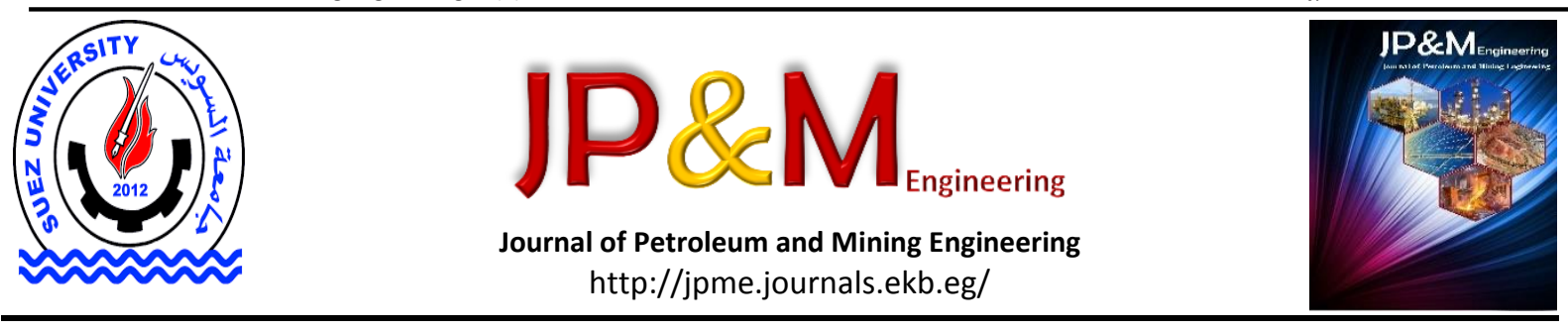

\title{
Minimizing Losses on Distribution System by PV and
}

\section{Compensating Capacitors}

\author{
El-Mahdy,A. A. ${ }^{1}$, Megahed, M.G. ${ }^{2}$, Seleym, A. ${ }^{2}$, Sweif, R. A. ${ }^{3}$, \\ Abdel-Salam, T. S. ${ }^{1}{ }^{3}$ \\ 1. Faculty of Energy and Environmental Engineering, The British University in Egypt, Cairo, \\ Egypt \\ 2. Faculty of Engineering, British university in Egypt, Cairo, Egypt \\ 3. Department of Electrical Engineering, Ain Shams University, Cairo, Egypt \\ *asmaa.ashraf@bue.edu.eg
}

\section{Article Info}

Received 10 Sept. 2019

Revised 24 Dec. 2019

Accepted 2 Feb. 2020

\section{Keywords}

Distributed generation; Cuckoo Search; Ant lion; Genetic algorithm; Power losses reduction; Voltage profile.

\begin{abstract}
Electric power has become an essential measure of the structure of modern society with most of today's daily activity based on the proposition that the desired electric power constantly exists. In the Electrical Distribution System (EDS), each component is essential to the process of distributing power from the site where it is generated then supplied to the customer who utilizes it. Distribution system plays an utmost role in providing the electrical energy from its generation point to the consumers' end by the use of transmission system. Since the $R / X-W h e r e ~ R$ is the Resistance and $X$ denotes for the impedance- ratio in the distribution network is high, the losses in the distribution system is more. In other words, since Electricity is so important, In addition, since there is losses in the networks where electricity is generated and transmitted to the end users, so it is typically wise to think of solutions to reduce such losses. One of the solutions is installing Distributed Generators (DGs) in the network. The DGs employed in this work is a mix of Photovoltaic panels (PVs) for active power compensation and capacitors for reactive power compensation. Location, range and type of capacitors used in the system manipulate the amount of compensation provided by the capacitors. To decide the location and size of the DGs, a load flow will be used and losses in the radial network are considered. The backward-forward sweep load flow technique is used on the IEEE 69 bus standard network. Also, the Genetic algorithm, antlion technique and Cuckoo search algorithm (CS) are used as optimization techniques. The results are tested on the IEEE 69-bus standard test network to validate the proposed algorithms. The proposed algorithms showed enhanced results compared to the genetic algorithms.
\end{abstract}

\section{Introduction}

The configuration of an Electrical Distribution System (EDS) is adjusted to diminish the real losses. Principally, the losses in an EDS arise due to two factors:

- Mistake in the network

- Burden on the feeders.

The losses taking place as a result of the overload or uneven load are dealt with in this paper. EDS is normally unevenly loaded and hereafter frequently require load balancing, which can also be done by reconfiguring the network using PV to substitute for active power and capacitors for reactive power.

Capacitor employment and network reconfiguration have been extensively employed to lessen power losses, and to maintain voltage profiles within allowable limits in distribution systems. Reconfiguration method proposed in this paper is based on loop elimination technique. In this method, the tie switches and sectionalized switches are 
operated, and network is reconfigured. The Optimal capacitor placement is carried out using Genetic Algorithm (GA). Furtherly, a united optimization algorithm is projected for merging network reconfiguration and capacitor placement. IEEE-69 bus test system and 85 standard bus structures are used as trials to make sure that the combined optimization technique and the loss reduction are computed and working successfully, the obtained results shows the effectiveness of the proposed method. The location of placing the capacitors in the distribution system is obligatory as of the load existing in the system [1]. There are many ways to reduce the active power losses of distribution network. Two important ways of solving the problem are:
- feeder reconfiguration, and

- $\quad$ capacitor placement.

The DG systems are constructed on a diversity of diverse technologies, which are founded on either renewables or fossil fuels sources. Solar, wind, geothermal and hydro-energies are some systems of those different technologies, which are founded on renewables. These technologies are more cost effectual for long-term strategies and more reliable with a smaller amount of environmental effects compared to the traditional centralized systems. Figure 1 demonstrates the diverse technologies for DG. It could be classified into Renewable or Nonrenewable technology.

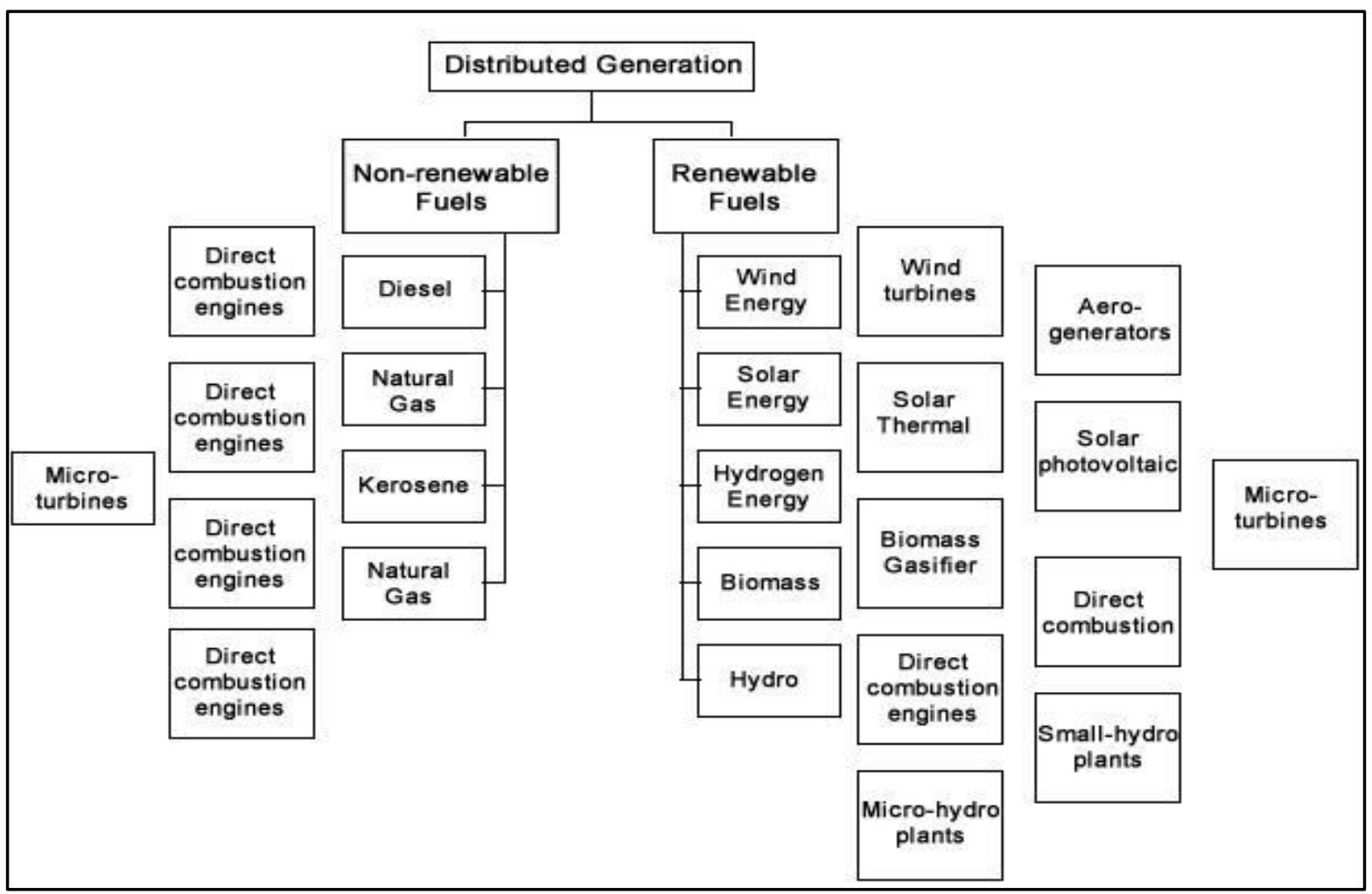

Figure 1 Distribution Generation technologie

In this thesis thesis, PV technology is considered as the distribution generation source. PV panels are used for whichever viable or local applications. They simply have the ability to convert light emissions into electricity. PV panels are made of smaller cells (range $<5 \mathrm{~kW}$ ) linked together creating a system of any size. The PV produces DC power output, which has to be changed into AC for consumers' to be able to use by the use of inverters. Even if PVs are environmentally friendly and have greater reliability with lowest care requirements, they are considered to be very costly. To avoid this high cost, cheaper components may be used in the industrial process. A PV cell is the basic building unit of PV modules and panels. The cells are prepared using doped silicon crystals. These building units are linked in series/parallel connections to build up the module or panel. Lastly, the PV arrays are formed by connecting those modules or panels to generate the electrical power. The basic maneuver of $\mathrm{PV}$ is that solar energy is taken from the sunlight by the PV cells. The sunlight's photons urge the electrons of the cells to become free and stream. Hence, it is converted to dc electrical power. Each cell offers from $2 \mathrm{~A}$ to $4 \mathrm{~A}$ and $0.5 \mathrm{~V}$ dependent on its size. In the array arrangements, cells are allied in series normally providing $12 \mathrm{~V}$ as the output voltage permitting battery charging. There are some restrictions such as the low generated power, high costs of plots or areas where PV systems placed and restrictions because of the geographic and weather deviations.[12]

Genetic Algorithm is selected to unravel the network reconfiguration issue as well as the capacitor placement issue. These two ways will be merged together to reach the best effect of reducing losses. This will be better than using one method on its own. The correctness of the obtained ways has been inspected by means of 2 standard distribution networks and results are presented in this work [1]. A suggested load flow technique is used for solving 
radial distribution systems using backward forward sweep technique. This method is demonstrated through 69-node test systems. The final test results reveal verifies that the suggested methods give optimal configuration with reduced losses [2].

The topic of harmonizing the load in distribution systems has gained an excessive amount of attention since the cost of electricity is so high, and as a result, a big amount of the current research on distribution automation has been directed to focus on the network reconfiguration for loss minimization and load balancing. Other than economic attentions, electric power loss is seen as the heat energy dissipated which rises the temperatures of the linked electric components and can result in insulation catastrophe on overburdening of the feeders. Unequal load balancing of the feeders may lead to overloading of some of the feeders. In practical systems, the method employed for reduction of losses and load balancing is network reconfiguration, which is the selection of the proper topological structure of the network for minimum load balancing index. [3]

To check the efficiency and the performance of the mentioned algorithms in choosing the ideal location and size of DGs or Capacitors in radial distribution power system, three test systems were used [4].

In this paper, IEEE 69-bus test systems are used to verify the proposed algorithms [5]. Excluding the base case, three groups of cases are considered while three different types of generation are used. [13]

- Group 1 using PV DGs only

- Group 2 using compensating capacitors only

- Group 3 using both compensating capacitors and PV DGs

Three different penetration levels of DG/capacitor placement are used for the different cases were:

$5 \% 10 \%, 15 \%, 20 \%, 25 \%$ and $30 \%$ of the total active/reactive power in both networks.

\section{Load Flow}

\section{Case Study}

Cuckoo are breathtaking birds, besides their beautiful sounds, they have an aggressive reproduction attitude.

We will first initiate by describing the reproduction performance of cuckoos and the features of L'evy flights of certain birds and fruit flies, then express the new CS, and how to implement it. Like other metaheuristic optimization procedures, it is an optimization that is inspired from nature. It is established upon the "obligate brood parasitism" of some cuckoo kinds in the environment which put their eggs in the nests of other birds. Those birds are called the host birds. Some Cuckoos have developed in a way that the lady parasitic Cuckoos can copy the colors and the pattern of the host eggs in order to not be revealed by the host eggs, thus the population of the
While studying distributed networks, the most important challenge faced is power-flow problem of the network. The power-flow equations are nonlinear, which makes it hard to use traditional optimization techniques.

Examining the power flow of a certain network in any radial distribution-system is considered to be the life-line of the network. It demonstrates the active and reactive power losses in each branch of the networks in addition to the demonstrations of the voltage magnitudes of each bus of the network under the steady state. So examining the load flow of any network is a must in order to plan, optimize and control the power system. To start with, analyzing the power flow is the first process to be finished taking into consideration whether the voltage-profiles are within the limits throughout the system or not. Moreover, in the operation process, load flow analysis is used to discover diverse arrangements to lessen the system's power-loss plus improving the voltageprofiles in the system. Several power flow methods are used for the transmission-systems like Gauss Seidel and Newton Raphson [6]. Throughout this paper, we are applying the load flow on a distribution network not a transmission network. Distribution networks are architecturally weakly meshed but wellthought-out as radially operating. These networks have the advantage of being simpler in design and cheaper in cost as a result. [14] The Backward-forward sweep technique is used. Once we know all currents and voltages everywhere in the network, one can easily calculate the power to be able to calculate the power losses. Next chapter will discuss the proposed algorithm to minimize power losses by adding DGs. The Cuckoo search algorithm will determine the size and location of the DGs.[15]

The algorithm is done on "MATLABC R2017b coding". Work is done on an A1278 Macbook $2.0 \mathrm{GHz}$ Core 2 Duo (P7350) 2GB RAM, 256 MB VRAM 160GB GHH storage and 8X DL "SuperDrive"

cuckoos increases. If under circumstances the host bird discovered that the eggs are not its own, it does action of two [7].

\section{- Throw the eggs away}

- Abandon the nest build new one

If it did not realize that the eggs are not of its own, once the first cuckoo egg hatches, it starts to blindly throw the other eggs out of the nest as it usually hatches earlier than the other eggs. This instinct action helps the cuckoo get the most share of food provided by the host. Not only that, studies had proven that the baby cuckoos can imitate the sound of the host babies to always gain more feeding chances. The Cuckoo Search which overemphasizes such breeding performance was propositioned by XinShe Yang and Suash Deb in 2009. From that time, the Cuckoo search algorithm technique has been applied widely to numerous engineering optimization 
problems like data blending in wireless networks [8], and to project a multi objective designs as consistent embedded system designs [8]. It was also combined with quantum computing principles [9], and with power series [10] to obtain better performance. In the $\mathrm{CS}$, each laid egg in a host's nest symbolizes a solution to our optimization problem, and a cuckoo egg

- Every cuckoo bird lay 1 egg separately, and place its egg in an arbitrary nest;

- The finest nests that have the high-standard eggs will continue to the subsequent generation;

- The number of existing host nests, $\mathrm{n}$ is fixed, and the cuckoo egg is exposed by the host bird probably by symbolizes a new solution. The goal is to replace the "not so good" solution "egg" with a better solution which is the "cuckoo's egg"in the nest. Figure 2 shows a flow chart explaining the steps of the cuckoo search optimization algorithm. A simple way to understand CS we assume that each nestonly one egg. The CS is assembled in 3 rules as follows:

$\mathrm{Pa} \in(0,1)$. When the host bird realizes that the egg is not hers, however it is the Cuckoos, this causes that some nests (eggs) will be thrown away as they are "worst nests" and as a result the related solutions will be rejected and thrown away from next calculations.

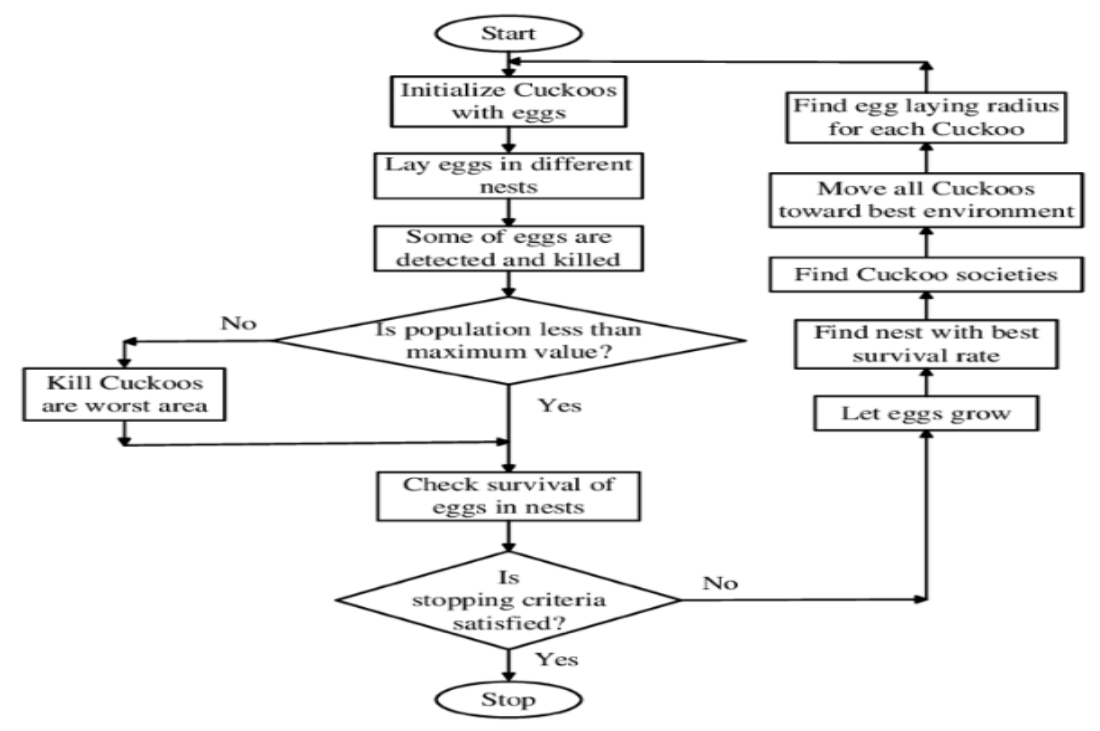

Figure 2 shows a flow chart explaining the steps of the cuckoo search optimization algorithm.

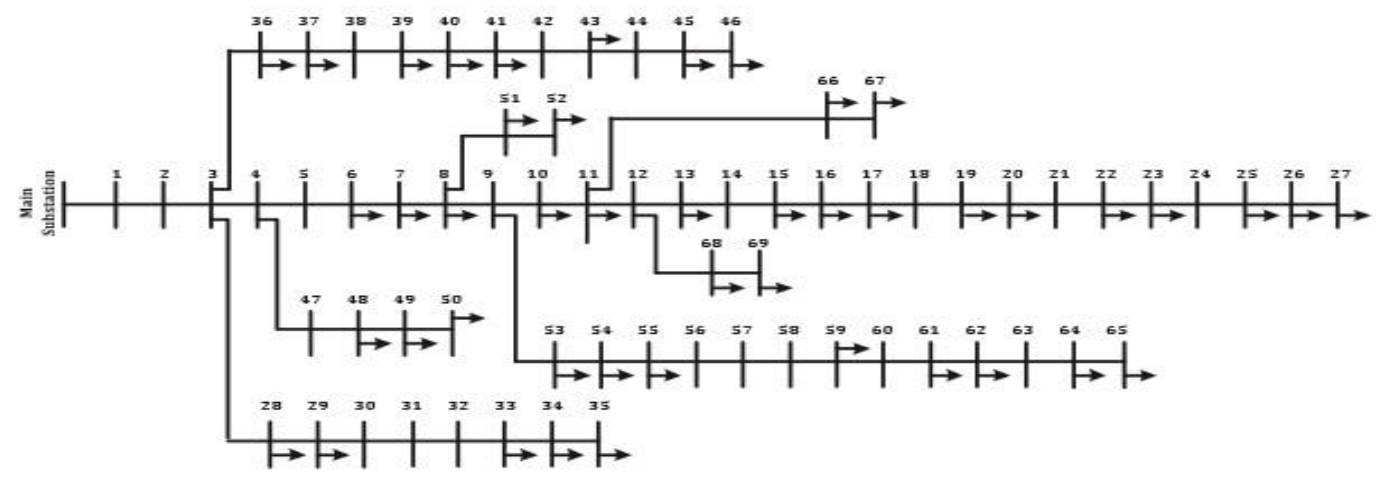

Figure 2 Single line diagram of IEEE 69 bus network

\section{Voltages per unit (p.u)}

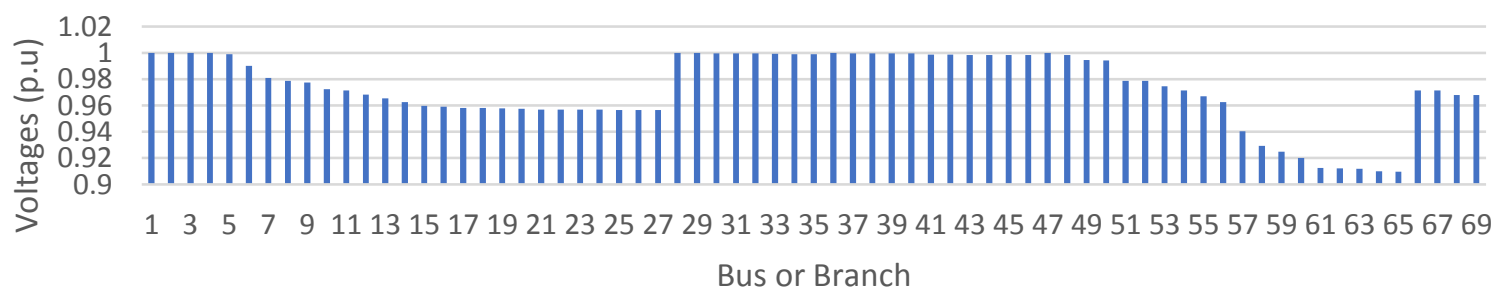

Figure 3 Node Voltage magnitudes of the base case of the IEEE 69-bus 


\section{Results}

This section will be divided into 2 sections. One for the load flow results after using the backwardforward technique that was proposed by SherMohammady [11] and showed reliable results. The other section will be a table of comparison between the GA in finding the size and location of the DGs, the antlion algorithm and the Cuckoosearch algorithm.

\section{1- Load Flow Results}

The Backward Forward Sweep algorithm load flow is executed for the "IEEE 69-Bus" network whose data is proposed in [11] and is shown in the Appendix. The overall loads of the network are $4.660 \mathrm{MW}$ which is

\section{Comparison between GA, CS and $A L$}

Table 1 shows a comparison between Different optimization techniques. The Genetic Algorithm, The antlion and the Cuckoo Search Algorithms. The Antlion and Cuckoo Search Algorithms have shared 3.80189MW active power and 2.6941MVAR reactive power).

The "IEEE 69-bus" network has 69 nodes and 68 branches. Figure 2 shows the 69-bus network Single line diagram with all 69 nodes and 68 branches. The 69-bus network has the following base values:

- Voltage magnitude $=12.66 \mathrm{KV}$

Base-apparent power $=100 \mathrm{MVA}$. The results display that the IEEE 69 bus has a weak voltage-profile from bus " 57 " to " 65 " due to the bus "61" high load.

The results reveal that:

All the bus voltages lie within the range of $(0.8713-$ 1) p.u.

The minimum-voltage $=0.8713$ p.u. at node " 54 "

The maximum branch current is 0.0404 p.u at the first branch very near values to those of the GA. The Antlion and CuckooSearch algorithms are verified and validated as Optimization techniques.

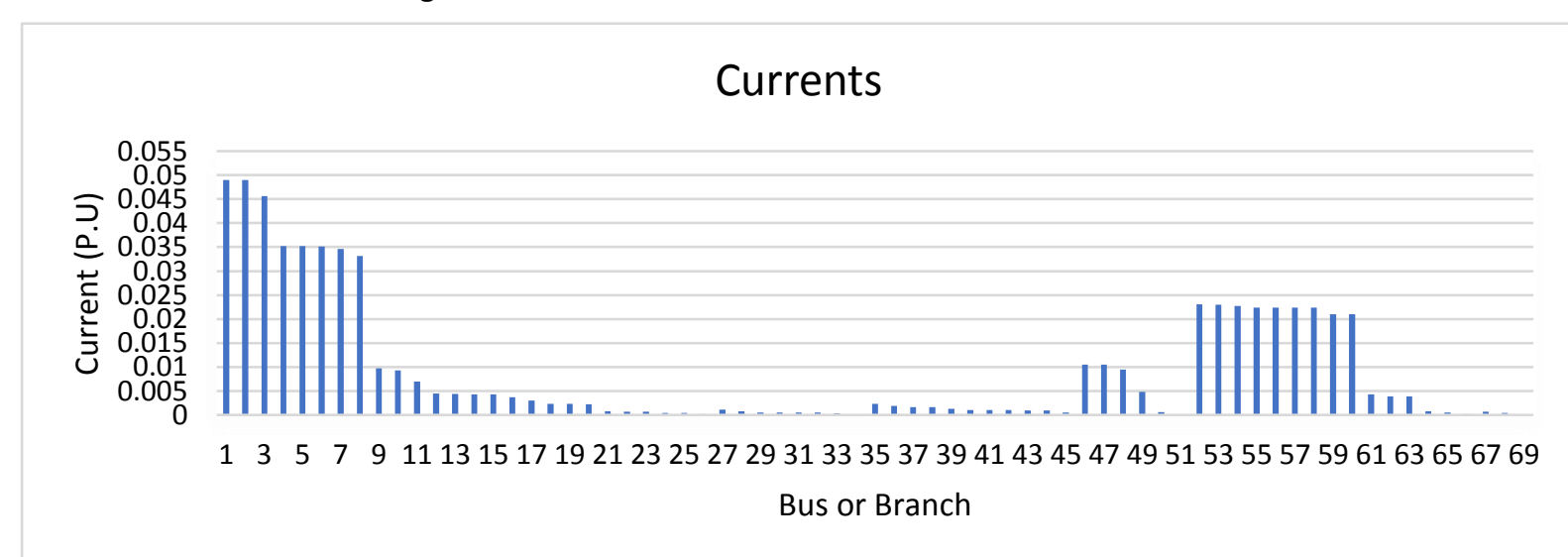

Figure 4 Branch current Magnitude of the base case of the IEEE 69-bus

\section{Conclusions}

The Genetic Algorithm (GA), Cuckoo search optimization algorithm and the Antlion Optimizer algorithm (ALO) techniques are presented in this paper to solve the problem of selecting the optimal size and location of DG placement in in the radial distribution system IEEE-69 bus system as a radial distribution system in order to minimize the total power losses. Backward/forward sweep load flow method is used for the load flow study of the radial distribution networks. The proposed DGs are compensating capacitors for improving the reactive power and using the PVs for enhancing the active power. The results prove validation of the applied algorithms GA, Cuckoo algorithm and antlion algorithm. The simulation results showed that the proposed algorithms are able to maximize the power losses reduction percentage.

\section{Acknowledgements}

The authors would also like to show their gratitude to the Prof. Tarek Saad Abdel-Salam and Dr. Rania Sweif for sharing their pearls of wisdom during this paper. 
Table 1 A comparison between different Optimization algorithms

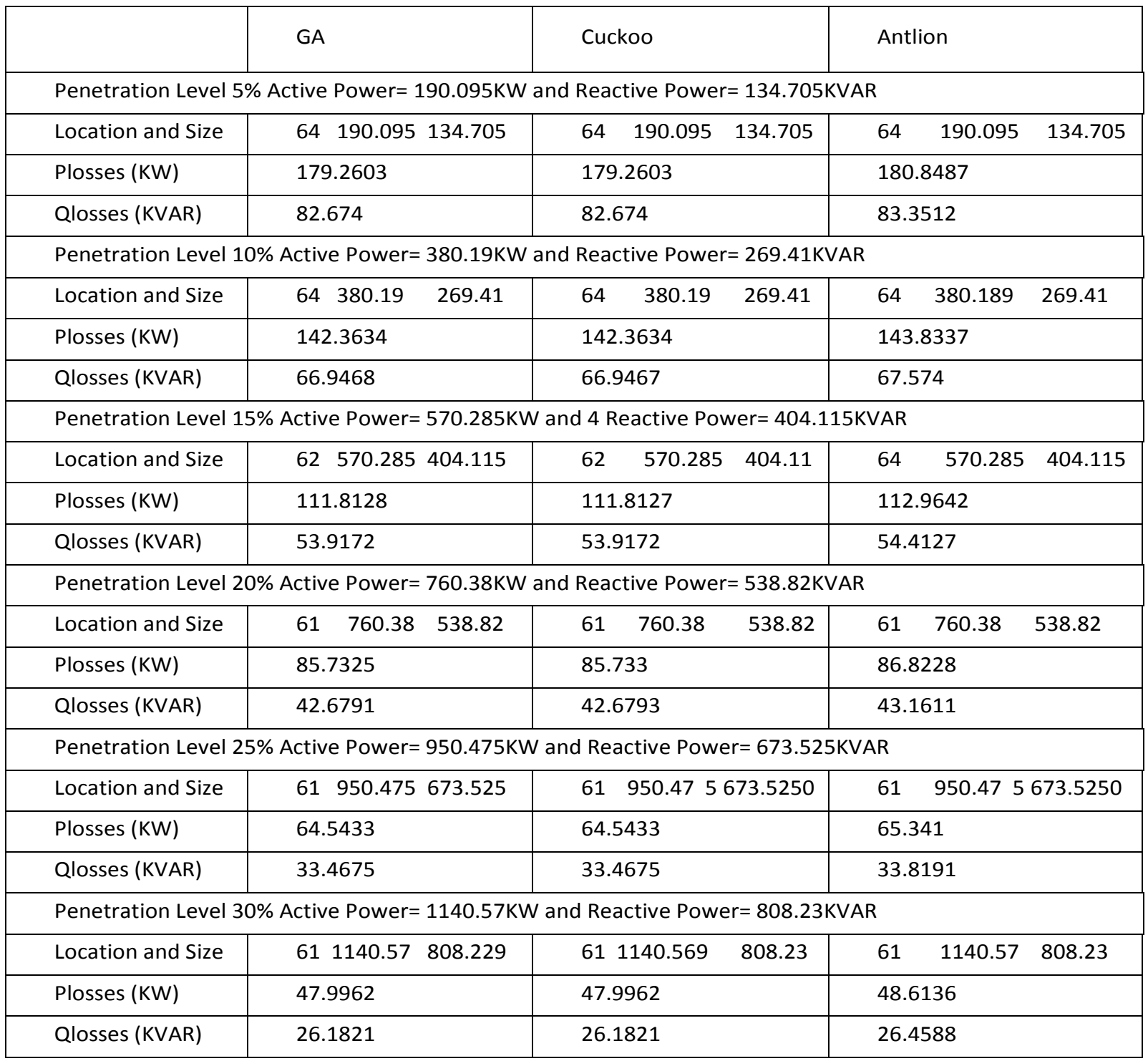

\section{References}

[1] [1] S. Segura, R. Romero, M.J. Rider Efficient heuristic algorithm used for optimal capacitor placement in distribution systems. Int J Electric Power Energy Syst, 32 (1) (2010), pp. 71-78.

[2] [2] D. B. Prakash, C. Lakshminarayana, "Optimal siting of capacitors in radial distribution network using Whale Optimization Algorithm", Alexandria Engineering Journal 56, 499-509 ElSevier, (2017).

[3] [3] Mohamed G. Z. Fouly, A. A. El-Mahdy, I. M. Mahmoud, Ashraf Seleym, R. A. Sweif, T. S. AbdelSalam "Power System Improvement using Different Al Optimization Techniques", The Future of Electricity Grids Challenges and opportunities Cigre Egypt, March 2019.

[4] [4] H. HassanzadehFard and A. Jalilian, "A novel objective function for optimal DG allocation in distribution systems using meta-heuristic algorithms", International Journal of Green Energy, vol. 13, no. 15, pp. 1615-1625, 2016.
[5] [5] S. Devi and M. Geethanjali, "Application of Modified Bacterial Foraging Optimization algorithm for optimal placement and sizing of Distributed Generation," Expert Systems with Applications, vol. 41, no. 6, pp. 2772-2781, 2014.

[6] [6] R. Prakash and B. Sujatha, "Optimal placement and sizing of DG for power loss minimization and VSI improvement using bat algorithm," 2016 National Power Systems Conference (NPSC), 2016.

[7] Available online 2 October 2018. https://www.mdpi.com/1996-1073/11/10/2547

[8] [7] R. B. Magadum and D. Kulkarni, "Power loss reduction by optimal location of DG using fuzzy logic," 2015 International Conference on Smart Technologies and Management for Computing, Communication, Controls, Energy and Materials (ICSTM), 2015.

[9] [8] S. Gopiya Naik, D. Khatod and M. Sharma, "Analytical approach for optimal siting and sizing of distributed generation in radial distribution 
networks", IET Generation, Transmission \& Distribution, vol. 9, no. 3, pp. 209-220, 2015.

[10] [9] A. Rezaee Jordehi, "Allocation of distributed generation units in electric power systems: A review", Renewable and Sustainable Energy Reviews, vol. 56, pp. 893-905, 2016.

[11] [10] Lei Zhang, Kaoshe Zhang and Gang Zhang, "Power distribution system reconfiguration based on genetic algorithm," 2016 IEEE Advanced Information Management, Communicates, Electronic and Automation Control Conference (IMCEC), Xi'an, 2016, pp. 80-84.

[12] [11] R. Al Abri, E. El-Saadany and Y. Atwa, "Optimal Placement and Sizing Method to Improve the Voltage Stability Margin in a Distribution System Using Distributed Generation", IEEE Transactions on Power Systems, vol. 28, no. 1, pp. 326-334, 2013.

[13] [12] R. A. Swief, T. S. Abdel-Salam, and Noha H. ElAmary, "Photovoltaic and Wind Turbine Integration Applying Cuckoo Search for Probabilistic Reliable Optimal Placement", Energies 2018, Vol. 11, Issue 1, 139. doi: 10.3390/en11010139. Available online 6 January 2018. http://www.mdpi.com/1996$1073 / 11 / 1 / 139$

[14] [13] Peter Makeen, R. A. Swief, T. S. Abdel-Salam, Noha H. El-Amary, "Smart Hybrid Micro-Grid
Integration for Optimal Power Sharing-Based Water Cycle Optimization Technique", Energies 2018, Vol. 11, Issue 5, 1083, 2018. doi: 10.3390/en11051083. Available online $27 \quad$ April 2018. http://www.mdpi.com/1996-1073/11/5/1083

[15] [14] Heba-Allah I. ElAzab, R. A. Swief, Noha H. ElAmary, and H. K. Temraz, "Unit Commitment Towards Decarbonized Network Facing Fixed and Stochastic Resources Applying Water Cycle Optimization", Energies 2018, Vol. 11, Issue 5, 1140, 2018. doi: 10.3390/en11051140. ISSN 19961073. Available online 3 May 2018. http://www.mdpi.com/1996-1073/11/5/1140

[16] [15] Heba-Allah I. ElAzab, R. A. Swief, Hanady H. Issa, Noha H. El-Amary, Alsnosy Balbaa, H. K. Temraz, "FPGA Eco Unit Commitment Based Gravitational Search Algorithm Integrating Plug-in Electric Vehicles", Energies 2018, Vol. 11, Issue 10, 2547, 2018. doi: 10.3390/en11102547. ISSN 19961073.

\section{Appendix: IEEE 69-bus Networks' Data}

In this Appendix, Table 2 shows the bus data for the "IEEE 69-bus system" while 3 shows the line data for "IEEE 69-bus system"

Table 269 Bus Data

\begin{tabular}{|c|c|c|c|c|c|}
\hline $\begin{array}{c}\text { Bus } \\
\text { Number }\end{array}$ & $\mathbf{P}(\mathbf{K W})$ & Q (KVAr) & $\begin{array}{c}\text { Bus } \\
\text { Number }\end{array}$ & $\mathbf{P}(\mathbf{K W})$ & Q (KVAr) \\
\hline 1 & O & O & 36 & 26 & 18.55 \\
\hline 2 & $\mathrm{O}$ & $\mathrm{O}$ & 37 & 26 & 18.55 \\
\hline 3 & $\mathrm{O}$ & 0 & 38 & 0 & $\mathrm{O}$ \\
\hline 4 & $\mathrm{O}$ & $\mathrm{O}$ & 39 & 24 & 17 \\
\hline 5 & 0 & $\mathrm{O}$ & 40 & 24 & 17 \\
\hline 6 & 2.6 & 2.2 & 41 & 1.2 & 1 \\
\hline 7 & 40.4 & 30 & 42 & $\mathrm{O}$ & $\mathrm{O}$ \\
\hline 8 & 75 & 54 & 43 & 6 & 4.3 \\
\hline 9 & 30 & 22 & 44 & $\mathrm{O}$ & $\mathrm{O}$ \\
\hline 10 & 28 & 19 & 45 & 39.22 & 26.3 \\
\hline 11 & 145 & 104 & 46 & 39.22 & 26.3 \\
\hline 12 & 145 & 104 & 47 & 0 & $\mathrm{O}$ \\
\hline 13 & 8 & 5 & 48 & 79 & 56.4 \\
\hline 14 & 8 & 5.5 & 49 & 384.7 & 274.5 \\
\hline 15 & $\mathrm{O}$ & $\mathrm{O}$ & 50 & 384.7 & 274.5 \\
\hline 16 & 45.5 & 30 & 51 & 40.5 & 28.3 \\
\hline 17 & 60 & 35 & 52 & 3.6 & 2.7 \\
\hline 18 & 60 & 35 & 53 & 4.35 & 3.5 \\
\hline 19 & $\mathrm{O}$ & $\mathrm{O}$ & 54 & 26.4 & 19 \\
\hline 20 & 1 & 0.6 & 55 & 24 & 17.2 \\
\hline 21 & 114 & 81 & 56 & 0 & 0 \\
\hline 22 & 5 & 3.5 & 57 & $\mathrm{O}$ & $\mathrm{O}$ \\
\hline 23 & $\mathrm{O}$ & $\mathrm{O}$ & 58 & $\mathrm{O}$ & $\mathrm{O}$ \\
\hline 24 & 28 & 20 & 59 & 100 & 72 \\
\hline 25 & O & $\mathrm{O}$ & 60 & O & 0 \\
\hline 26 & 14 & 10 & 61 & 1244 & 888 \\
\hline 27 & 14 & 10 & 62 & 32 & 23 \\
\hline 28 & 26 & 18.6 & 63 & $\mathrm{O}$ & 0 \\
\hline 29 & 26 & 18.6 & 64 & 227 & 162 \\
\hline 30 & 0 & $\mathrm{O}$ & 65 & 59 & 42 \\
\hline 31 & $\mathrm{O}$ & 0 & 66 & 18 & 13 \\
\hline 32 & $\mathrm{O}$ & 0 & 67 & 18 & 13 \\
\hline 33 & 14 & 10 & 68 & 28 & 20 \\
\hline 34 & 19.5 & 14 & 69 & 28 & 20 \\
\hline 35 & 6 & 4 & & & \\
\hline
\end{tabular}


Table 3 line Data for bus 69 system

\begin{tabular}{|c|c|c|c|c|c|c|c|}
\hline From & To & R(ohms) & $\mathbf{X}(\mathrm{ohms})$ & From & To & R(ohms) & X(ohms) \\
\hline 1 & 2 & 0.0005 & 0.0012 & 3 & 36 & 0.0044 & 0.0108 \\
\hline 2 & 3 & 0.0005 & 0.0012 & 36 & 37 & 0.064 & 0.1565 \\
\hline 3 & 4 & 0.0015 & 0.0036 & 37 & 38 & 0.1053 & 0.123 \\
\hline 4 & 5 & 0.0251 & 0.0294 & 38 & 39 & 0.0304 & 0.0355 \\
\hline 5 & 6 & 0.366 & 0.1864 & 39 & 40 & 0.0018 & 0.0021 \\
\hline 6 & 7 & 0.381 & 0.1941 & 40 & 41 & 0.7283 & 0.8509 \\
\hline 7 & 8 & 0.0922 & 0.047 & 41 & 42 & 0.31 & 0.3623 \\
\hline 8 & 9 & 0.0493 & 0.0251 & 42 & 43 & 0.041 & 0.0478 \\
\hline 9 & 10 & 0.819 & 0.2707 & 43 & 44 & 0.0092 & 0.0116 \\
\hline 10 & 11 & 0.1872 & 0.0619 & 44 & 45 & 0.1089 & 0.1373 \\
\hline 11 & 12 & 0.7114 & 0.2351 & 4 & 46 & 0.0009 & 0.0012 \\
\hline 12 & 13 & 1.03 & 0.34 & 46 & 47 & 0.0034 & 0.0084 \\
\hline 13 & 14 & 1.044 & 0.345 & 47 & 48 & 0.0851 & 0.2083 \\
\hline 14 & 15 & 1.058 & 0.3496 & 48 & 49 & 0.2898 & 0.7091 \\
\hline 15 & 16 & 0.1966 & 0.065 & 49 & 50 & 0.0822 & 0.2011 \\
\hline 16 & 17 & 0.3744 & 0.1238 & 8 & 51 & 0.0928 & 0.0473 \\
\hline 17 & 18 & 0.0047 & 0.0016 & 51 & 52 & 0.331 & 0.1114 \\
\hline 18 & 19 & 0.3276 & 0.1083 & 9 & 53 & 0.174 & 0.0886 \\
\hline 19 & 20 & 0.2106 & 0.069 & 53 & 54 & 0.203 & 0.1034 \\
\hline 20 & 21 & 0.3416 & 0.1129 & 54 & 55 & 0.2842 & 0.1447 \\
\hline 21 & 22 & 0.014 & 0.0046 & 55 & 56 & 0.2813 & 0.1433 \\
\hline 22 & 23 & 0.1591 & 0.0526 & 56 & 57 & 1.59 & 0.5337 \\
\hline 23 & 24 & 0.3463 & 0.1145 & 57 & 58 & 0.7837 & 0.263 \\
\hline 24 & 25 & 0.7488 & 0.2475 & 58 & 59 & 0.3042 & 0.1006 \\
\hline 25 & 26 & 0.3089 & 0.1021 & 59 & 60 & 0.3861 & 0.1172 \\
\hline 26 & 27 & 0.1732 & 0.0572 & 60 & 61 & 0.5075 & 0.2585 \\
\hline 3 & 28 & 0.0044 & 0.0108 & 61 & 62 & 0.0974 & 0.0496 \\
\hline 28 & 29 & 0.064 & 0.1565 & 62 & 63 & 0.145 & 0.0738 \\
\hline 29 & 30 & 0.3978 & 0.1315 & 63 & 64 & 0.7105 & 0.3619 \\
\hline 30 & 31 & 0.0702 & 0.0232 & 64 & 65 & 1.041 & 0.5302 \\
\hline 31 & 32 & 0.351 & 0.116 & 11 & 66 & 0.2012 & 0.0611 \\
\hline 32 & 33 & 0.839 & 0.2816 & 66 & 67 & 0.0047 & 0.0014 \\
\hline 33 & 34 & 1.708 & 0.5646 & 12 & 68 & 0.7394 & 0.2444 \\
\hline 34 & 35 & 1.474 & 0.4873 & 68 & 69 & 0.0047 & 0.0016 \\
\hline
\end{tabular}

